\title{
Contribution of the Determination of the Load on Suspension Ring of the Underframe of the Hydraulic Excavator
}

\author{
Slaviša Salinic ${ }^{1 *}$, Marko Nikolic ${ }^{1}$, Goran Boskovic ${ }^{1}$ \\ ${ }^{1}$ Faculty of Mechanical and Civil Engineering, University of Kragujevac, 36000 Kraljevo (Serbia)
}

In this paper it is presented a method for determining the load on the suspension ring of underframe of hydraulic excavator which binds radial-axial bearing. The approach is based on the use of Kane's equations with undetermined multipliers of constraints. The expressions are derived in symbolic form for the forces which suspension ring is exposed during the operation of digging. In addition to the kinematic and inertial parameters of the excavator, in these expressions are included the forces in hydro cylinders as well as parameters that characterize the operation of digging. For specific numerical values of the system parameters, numerical analysis is carried out and the appropriate load graphics are presented.

Keywords: Hydraulic excavator, Suspension ring, Multibody, Dynamics

\section{INTRODUCTION}

Excavators are universal construction machines with cyclic work, which primary task is excavation of soil, and the secondary is transport of excavation to the place of disposal or loading in appropriate transportation means. Their working cycle consists of: digging of soil with the bucket filling, the bucket lifting, transfer of excavated material to the place of discharge, bucket discharge and taking up the original position.

Excavator (Figure 1) consists of the basic machine and excavation device. The basic machine consists of running gear device and rotating part of machine, while the excavator device is composed of boom, bucket stick and bucket. Depending on type of the running gear device there are two types of excavators: wheel excavators and crawler excavators which are most common in the application.

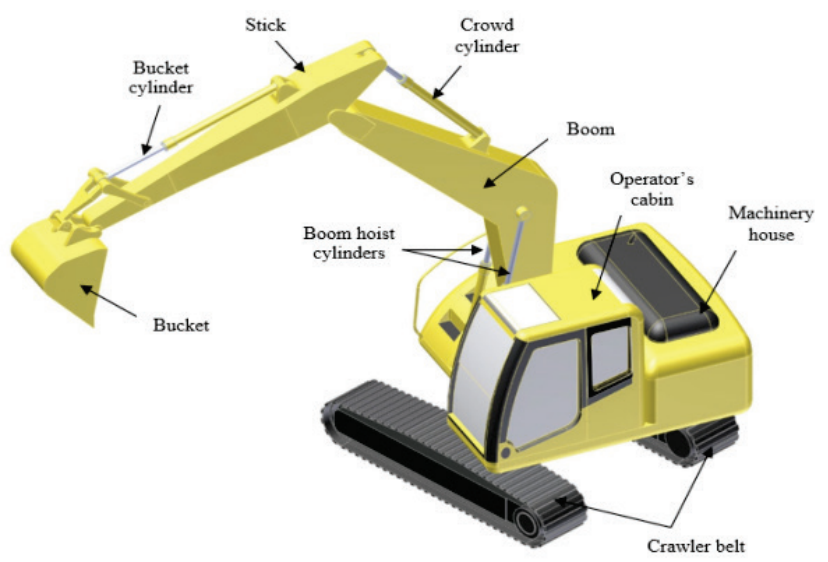

Figure 1: The basic elements of a hydraulic excavator with caterpillar tracks
Crawler excavators are self-propelled machines, whose running gear device allows moving the excavator from one to another place of excavation, while not performing transport of excavated material. The running gear device of excavator consists of frame, caterpillar running gear machine and mechanism for drive and braking. Caterpillar tracks have independent drive by individual hydraulic motors by a system mechanical transmission,thereby providing a synchronized or separated movement of the caterpillar tracks.

Rotating platform is the basic metal construction of the excavator, on which are mounted working device, hydraulic drive, cabin with driving system and rotating mechanism. The main objective of placement of devices on rotating platform is achievement of best static moment, by which it prevents the overturning of the excavator. For that reason, on rotary platform is placed the counterweight. Rotating platform with rotating-supporting ring is connected with running gear machine, and thus own and working loads that acting on working device during operation, are transferred to the ground.

The underframe is integral part of the running gear device of the excavator and it represents one of the most important parts of the supporting structure of the excavator.

Its main task is to transfer the load from the upper frame, by slewing bearing on mechanism for movement. Support ring respresent a part of underframe which binds to slewing bearing. It is usually welded structure made from sheet metal, whose elements are made by cutting and folding. 


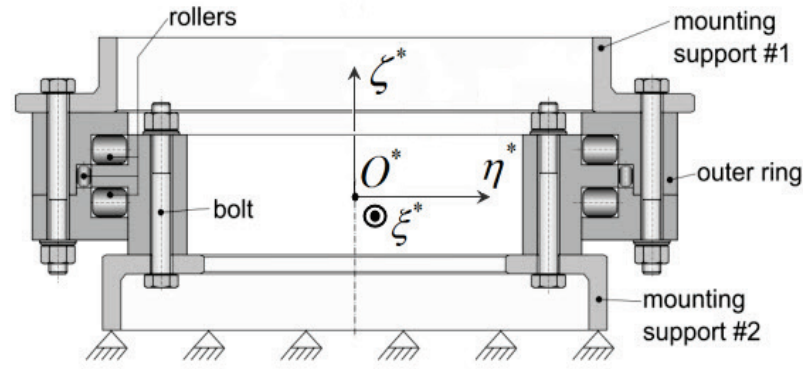

Figure 2: Connection of slewing bearing, suspension ring and rotating platform

Besides transferring the load, slewing bearing have task to ensure the stability and to allow undisturbed functioning of the rotating part of excavator. This bearing is made in the form of one or more rows of balls or rollers, with gearing on the bearing ring which is connected to support ring by bolts (Figure 2). Bearing is usually composed of three rings, between which are mounted rolling elements (balls, rollers or combined).

Loads that will be defined and determined in this paper will have multiple functions in future research:

- More precise calculation of bearing loading and selecting it on the basis of calculations

- Calculation of bearing life in accordance with exploitation parameters

- Possibilities of further structural development of support ring and modification of underframe construction

- Possibilities of detailed calculations of the required lubrication parameters

\section{HYDRAULIC EXCAVATOR KINEMATIC RELATIONS}

In Figure 3, the multibody meodel of a hydraulic excavator is showen [1]. The bodies $\left(V_{i}\right) \quad(i=0,1, \ldots, 4)$ represents, respectively, chassis with caterpillars, roboting platform with driver's cab, boom, stick, and bucket. The considerations in the paper are based on the assumptions of rigid soil foundation and immovable caterpillars. The motion of the excavator with respect to the fixed inertial reference frame $O x y z$ is described by the generalised coordinates $q_{i}(i=1, \ldots, 4)$. At that, the vertical $z$ axis is directed upwards and the $x$ axis represents the axis of material symmetry of body $\left(V_{0}\right)$. The coordinate $q_{i}$ represents the relative rotation of body $\left(V_{i}\right)$ with respect to $\left(V_{i-1}\right)$ carried out about the joint axis determined by the unit vector $\boldsymbol{e}_{i}$ fixed to the body $\left(V_{i-1}\right)$. The local coordinate frames $C_{0} \xi_{0} \eta_{0} \zeta_{0}$ and $O_{i} \xi_{i} \eta_{i} \zeta_{i} \quad(i=1, \ldots, 4)$ are fixed to bodies $\left(V_{i}\right)(i=0,1, \ldots, 4)$, respectively, in a manner shown in Figure 3. For the purposes of the further considerations, let us introduce a coordinate frame $O^{*} \xi^{*} \eta^{*} \zeta^{*}$ fixed to body $\left(V_{0}\right)$ at point $O^{*}$ representing the center of the slewing bearing. The vectors $\boldsymbol{e}_{\lambda^{*}}, \boldsymbol{e}_{\mu^{*}}$, and $\boldsymbol{e}_{v^{*}}$ denote the unit vectors of the axes $\xi^{*}, \eta^{*}$, and $\zeta^{*}$, respectively.

Without loss of generality, it is assumed that the configuration $q_{1}=0, q_{2}=0, q_{3}=0, q_{4}=0$ is a reference configuration of the excavator and that, in this configuration, the axes of all local coordinate frames are parallel to the corresponding axes of the inertial reference frame, that is, $\xi_{i} \square x, \eta_{i} \square y, . \zeta_{i} \square z$.

The mass centres of bodies $\left(V_{i}\right)(i=0,1, \ldots, 4)$ are denoted by $C_{i}(i=0,1, \ldots, 4)$. According to $[2,3]$, the transformation matrix $\boldsymbol{A}_{i, j}(i=0, \ldots, 4 ; j=1, \ldots, 4)$ from $O_{j} \xi_{j} \eta_{j} \zeta_{j}$ to $O_{i} \xi_{i} \eta_{i} \zeta_{i}$ reference frames $(i=0$ corresponds to the frame $C_{0} \xi_{0} \eta_{0} \zeta_{0}$ ) has the form:

$$
\begin{aligned}
& \boldsymbol{A}_{i . j}=\prod_{k=i+1}^{j} \boldsymbol{A}_{k}^{r}=\prod_{k=i+1}^{j}\left[\boldsymbol{I}+\left(1-\cos q_{k}\right)\left(\tilde{\boldsymbol{e}}_{k}^{(k)}\right)^{2}+\right. \\
& \left.+\tilde{\boldsymbol{e}}_{k}^{(k)} \sin q_{k}\right], i<j,
\end{aligned}
$$

where $\boldsymbol{A}_{k}^{r} \in R^{3 \times 3}$ is the Rodriguez matrix [2], $\boldsymbol{I} \in R^{3 \times 3}$ is the identity matrix, and $\tilde{\boldsymbol{e}}_{k}^{(k)} \in R^{3 \times 3}$ is the skew -

symmetric matrix $[2,4]$ associated with the vector $\boldsymbol{e}_{k}^{(k)}$. In further considerations the right superscript $(k)$ indicates that

components of the corresponding vectors and matrices are given in the $O_{k} \xi_{k} \eta_{k} \zeta_{k}$ local frame. In regard to [5,6], the following kinematic relations of the considering hydraulic excavator hold [1]:

$$
\begin{aligned}
& \boldsymbol{\omega}_{i}^{(i)}=\boldsymbol{A}_{i-1, i}^{T} \boldsymbol{\omega}_{i-1}^{(i-1)}+\dot{q}_{i} \boldsymbol{e}_{i}^{(i)}, i=1, \ldots, 4 \\
& \boldsymbol{\varepsilon}_{i}^{(i)}=\boldsymbol{A}_{i-1, i}^{T} \boldsymbol{\varepsilon}_{i-1}^{(i-1)}+\ddot{q}_{i} \boldsymbol{e}_{i}^{(i)}+\dot{q}_{i} \boldsymbol{A}_{i-1, i}^{T} \tilde{\boldsymbol{\omega}}_{i-1}^{(i-1)} \boldsymbol{e}_{i}^{(i-1)}, i=1, \ldots, 4 \\
& \boldsymbol{V}_{C_{i}}^{(i)}=\boldsymbol{A}_{i-1, i}^{T}\left(\boldsymbol{V}_{C_{i-1}^{(i-1)}}+\tilde{\boldsymbol{\omega}}_{i-1}^{(i-1)}\left(\boldsymbol{l}_{i-1}^{(i-1)}-\boldsymbol{l}_{C_{i-1}^{(i-1)}}\right)\right)+\tilde{\boldsymbol{\omega}}_{i}^{(i)} \boldsymbol{l}_{C_{i}}^{(i)}, \\
& i=1, \ldots, 4 \\
& \boldsymbol{a}_{C_{i}}^{(i)}=\boldsymbol{A}_{i-1, i}^{T}\left(\boldsymbol{a}_{C_{i-1}^{(i-1)}}+\tilde{\boldsymbol{\varepsilon}}_{i-1}^{(i-1)}\left(\boldsymbol{l}_{i-1}^{(i-1)}-\boldsymbol{l}_{C_{i-1}^{(i-1)}}^{(i)}\right)+\right. \\
& \left.\left(\tilde{\boldsymbol{\omega}}_{i-1}^{(i-1)}\right)^{2}\left(\boldsymbol{l}_{i-1}^{(i-1)}-\boldsymbol{l}_{C_{i-1}^{(i-1)}}\right)\right)+\tilde{\boldsymbol{\varepsilon}}_{i}^{(i)} \boldsymbol{l}_{C_{i}}^{(i)}+\left(\tilde{\boldsymbol{\omega}}_{i}^{(i)}\right)^{2} \boldsymbol{l}_{C_{i}}^{(i)}, \\
& i=1, \ldots, 4
\end{aligned}
$$

where $\boldsymbol{\omega}_{i}, \boldsymbol{\varepsilon}_{i}, \boldsymbol{V}_{C_{i}}$ and $\boldsymbol{a}_{C_{i}}$ are, respectively, the angular velocity, the angular accelerations, the velocity of the mass centre $C_{i}$ and the acceleration of the mass centre of body $\left(V_{i}\right)$, and where $l_{i}=\left|\overrightarrow{O_{i} O_{i+1}}\right|(i=1, \ldots, 3), \quad l_{0}=\left|\overrightarrow{C_{0} O_{1}}\right|$, $l_{C_{i}}=\left|\overrightarrow{O_{i} C_{i}}\right|(i=1, \ldots, 4)$, and $l_{C_{0}}=[0,0,0]^{T}$.

Since the body $\left(V_{0}\right)$ is immovable, the following holds:

$\boldsymbol{V}_{C_{0}}^{(0)}=[0,0,0]^{T}, \quad \boldsymbol{a}_{C_{0}}^{(0)}=[0,0,0]^{T}$. 


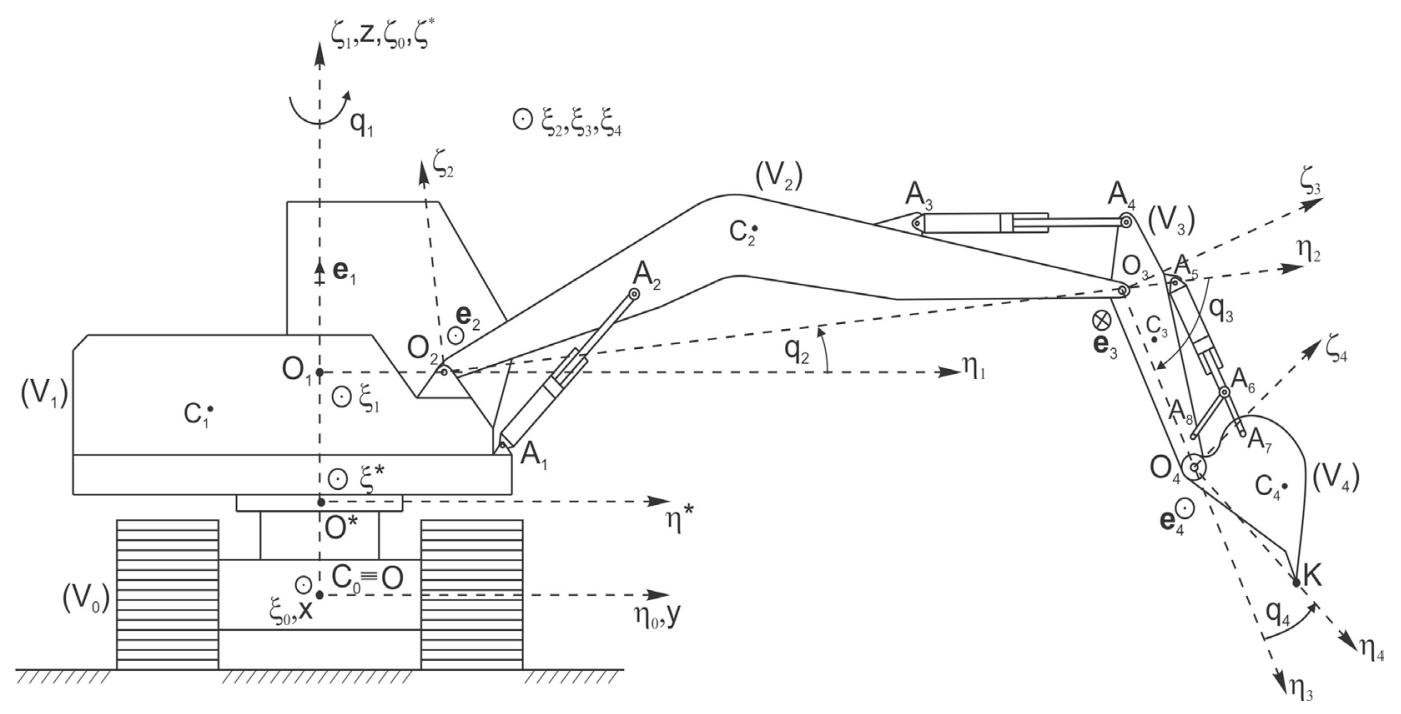

Figure 3: Multibody model of a hydraulic excavator

\section{DETERMINATION OF THE LOAD ON SUSPENSION RING OF THE UNDERFRAME DURING THE DIGGING TRANSPORTATION TASK}

The interaction between the bucket and the soil during the excavation phase is shown in Figure 4. The digging force $\boldsymbol{F}_{W}$ acts on the centre $K$ of the cutting edge of the bucket. The force $\boldsymbol{F}_{W}$ depends on various factors such as the depth of the bucket tip K, the width of the bucket, the terrain slope, and the soil physical characteristics. Different expressions for the magnitude $\mathrm{F}_{W}$ of the force $\boldsymbol{F}_{W}$ can be found in $[7,8,9,10]$. The digging angle is denoted by $\theta_{d g}$ and $\theta_{b}$ represents the angle between the bucket bottom and the $\eta_{4}$ - axis. In regard to $[7,11]$, the angle $\delta$ varies in the interval $0,1 \leq \delta \leq 0,45$ and depends on the digging angle, digging condition, and the wear of the bucket cutting edge. As in $[7,11,12]$, in this paper it is taken that this angle is constant and equal to $\delta=0,1$.

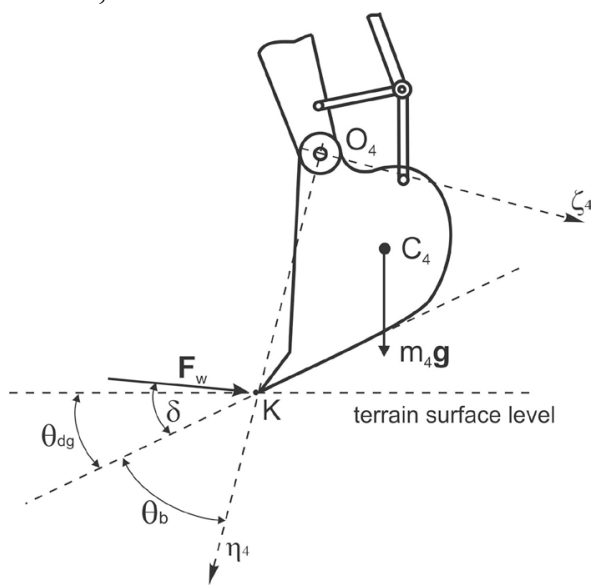

Figure 4: Interaction between the bucket and the soil

In accordance with Figure 4, the force $\boldsymbol{F}_{W}$ can be written as

$\boldsymbol{F}_{W}^{(4)}=\left[0,-\mathrm{F}_{W} \cos \left(\delta+\theta_{b}\right), \mathrm{F}_{W} \sin \left(\delta+\theta_{b}\right)\right]^{T}$.
The moment of the force $\boldsymbol{F}_{W}$ relative to point $C_{4}$ is determined by the following expression:

$$
\boldsymbol{M}_{4}^{(4)}=-\tilde{\boldsymbol{F}}_{W}^{(4)}\left({\overrightarrow{O_{4} K}}^{(4)}-\boldsymbol{l}_{C_{4}}^{(4)}\right) .
$$

Hence, the external force system exerted on bucket can be represented by a force system consisting of a force equal to $\boldsymbol{F}_{W}$ that passing through the mass centre $C_{4}$, the gravity force $m_{4} g$ of the bucket, and a couple with torque $\boldsymbol{M}_{4}$.

Based on approach from [14], the load of the suspension ring can be represented by a force passing through the point $O^{*}$ :

$\boldsymbol{R}^{*}=\left[\lambda_{1}, \lambda_{2}, \lambda_{3}\right]^{T}$

and a couple with torque

$\boldsymbol{M}^{*}=\left[\lambda_{4}, \lambda_{5}, 0\right]^{T}$

where $\lambda_{i}(i=1, \ldots, 5)$ are the projections of the vectors $\boldsymbol{R}^{*}$ and $\boldsymbol{M}^{*}$ onto the corresponding axes of the frame $O^{*} \xi^{*} \eta^{*} \zeta^{*}$. Based on [14], these projections are determined by the following expressions:

$$
\begin{aligned}
& \lambda_{r}=\sum_{p=1}^{4}\left[\boldsymbol{F}_{p}^{(p) T} \boldsymbol{b}_{p, r}^{V(p)}+\left(\boldsymbol{M}_{p}^{(p)}\right)^{T} \boldsymbol{b}_{p, r}^{\omega(p)}\right]- \\
& -\sum_{p=1}^{4} m_{p}\left(\boldsymbol{a}_{C_{p}}^{(p)}\right)^{T} \boldsymbol{b}_{p, r}^{V(p)}- \\
& -\sum_{p=1}^{4}\left(\boldsymbol{I}_{C_{p}} \boldsymbol{\varepsilon}_{p}^{(p)}+\tilde{\boldsymbol{\omega}}_{p}^{(p)} \boldsymbol{I}_{C_{p}} \boldsymbol{\omega}_{p}^{(p)}\right)^{T} \boldsymbol{b}_{p, r}^{\omega(p)}, r=1, \ldots, 5
\end{aligned}
$$

where it is taken that an external force system exerted on body $\left(V_{p}\right)(p=1, \ldots, 3)$ is represented by an equivalent force system consisting of a force $\boldsymbol{F}_{p}$ passing through the mass centre $C_{p}$ together with a couple with torque $\boldsymbol{M}_{p}$. In Eq. (11), $\boldsymbol{I}_{C_{p}}$ represents the centroidal inertia tensor of the body $\left(V_{p}\right)$ expressed in the local frame $C_{p} \xi_{p} \eta_{p} \zeta_{p}$ whose axes are chosen so that $C_{p} \xi_{p} \square O_{p} \xi_{p}$, $C_{p} \eta_{p} \square O_{p} \eta_{p}$ and $C_{p} \zeta_{p} \square O_{p} \zeta_{p}$ hold. 
Taking this into account, the projections of vectors in both coordinate frames $C_{p} \xi_{p} \eta_{p} \zeta_{p}$ and $O_{p} \xi_{p} \eta_{p} \zeta_{p}$ are the same.

Based on the considerations in [14], the vectors $\boldsymbol{b}_{p, r}^{\omega(p)}$ and $\boldsymbol{b}_{p, r}^{V(p)}$ are determinined by the following expressions:

$\boldsymbol{b}_{p, r}^{\omega(p)}=\left\{\begin{array}{l}{[0,0,0]^{T}, r=1,2,3 ; p=1, \ldots, 4} \\ \boldsymbol{A}_{0, p}^{T}[1,0,0]^{T}, r=4 ; p=1, \ldots, 4 \\ \boldsymbol{A}_{0, p}^{T}[0,1,0]^{T}, r=5 ; p=1, \ldots, 4\end{array}\right.$

$$
\boldsymbol{b}_{p, r}^{V(p)}=\left\{\begin{array}{c}
\boldsymbol{A}_{0, p}^{T}[1,0,0]^{T}, r=1 ; p=1, \ldots, 4 \\
\boldsymbol{A}_{0, p}^{T}[0,1,0]^{T}, r=2 ; p=1, \ldots, 4 \\
\boldsymbol{A}_{0, p}^{T}[0,0,1]^{T}, r=3 ; p=1, \ldots, 4 \\
\tilde{\boldsymbol{e}}_{\lambda^{*}}^{(1)}\left({\overrightarrow{O^{*} O_{1}}}^{(1)}+\boldsymbol{l}_{c_{1}}^{(1)}\right), r=4 ; p=1 \\
\tilde{\boldsymbol{e}}_{\mu^{*}}^{(1)}\left({\overrightarrow{O^{*} O_{1}}}^{(1)}+\boldsymbol{l}_{c_{1}}^{(1)}\right), r=5 ; p=1 \\
\left.\tilde{\boldsymbol{e}}_{\lambda^{*}\left(\boldsymbol{A}_{1, p}^{T}\right.}{\overrightarrow{O^{*} O_{1}}}^{(1)}+\boldsymbol{l}_{c_{p}}^{(p)}+\sum_{j=1}^{p-1} \boldsymbol{A}_{j, p}^{T} \boldsymbol{l}_{j}^{(j)}\right), r=4 ; p>1 \\
\tilde{\boldsymbol{e}}_{\mu^{*}(p)}\left(\boldsymbol{A}_{1, p}^{T}{\overrightarrow{O^{*} O_{1}}}^{(1)}+\boldsymbol{l}_{c_{p}}^{(p)}+\sum_{j=1}^{p-1} \boldsymbol{A}_{j, p}^{T} \boldsymbol{l}_{j}^{(j)}\right), r=5 ; p>1
\end{array}\right.
$$

where:

$\left.\begin{array}{l}\boldsymbol{e}_{\lambda^{*}}^{(p)}=\boldsymbol{A}_{0, p}^{T}[1,0,0]^{T} \\ \boldsymbol{e}_{\mu^{*}}^{(p)}=\boldsymbol{A}_{0, p}^{T}[0,1,0]^{T} \\ \overrightarrow{O^{*} O_{1}}(1)=\left[0,0, \overline{O^{*} O_{1}}\right]^{T}\end{array}\right\}$.

\section{NUMERICAL EXAMPLE}

For purposes of determining the numerical values of projections $\lambda_{i}(i=1, \ldots, 5)$ the following values of the excavator parameters are used ( see [7,11,12]): $m_{1}=6420 \mathrm{~kg}, m_{2}=1566 \mathrm{~kg}, m_{3}=735 \mathrm{~kg}, m_{4}=432 \mathrm{~kg}$, $I_{C_{2} \xi_{2}}=14250.6 \mathrm{~kg} \mathrm{~m}^{2}, \quad I_{C_{3} \xi_{3}}=727.7 \mathrm{~kg} \mathrm{~m}^{2}$, $I_{C_{4} \xi_{4}}=224.6 \mathrm{~kg} \mathrm{~m}^{2}, \quad l_{1}=0.05 \mathrm{~m}, \quad l_{2}=5.16 \mathrm{~m}$, $l_{3}=2.59 \mathrm{~m}, \quad \overline{O_{4} K}=1.33 \mathrm{~m}, \quad \overline{O^{*} O_{1}}=0.76 \mathrm{~m}$, $l_{C_{1}}=0.61 \mathrm{~m}, l_{C 2}=2.71 \mathrm{~m}, \quad l_{C 3}=0.64 \mathrm{~m}, \quad l_{C 4}=0.65 \mathrm{~m}$, $\gamma_{4}=1.92, \quad \angle\left(\boldsymbol{l}_{C_{1}}, \eta_{1}\right)=3.49305, \quad \angle\left(\boldsymbol{l}_{C 2}, \eta_{2}\right)=0.2566$, $\angle\left(\boldsymbol{l}_{C 3}, \eta_{3}\right)=0.3316, \angle\left(\boldsymbol{l}_{C 4}, \eta_{4}\right)=0.3944, \theta_{b}=1.0472$. The quantities $I_{C_{i}} \xi_{i}(i=2,3,4)$ represent second-order inertial moments about the axes through the gravity centres $C_{p} \xi_{p}(p=2,3,4)$, respectively. At that, as in [13], it is taken that the time interval of the considered digging task reads $0 \leq t \leq 3 s$ and that:

$q_{1}(t) \equiv 0$,

$q_{2}(t) \equiv-0.1744$, $q_{3}(t) \equiv 0.436$,

$q_{4}(t)=-0.1744 t^{3}-0.7848 \mathrm{t}^{2}$

and

$$
F_{w}(t)=2.1812 t^{3}-18.097 t^{2}+35.9936 t[k N] \text {. }
$$

The external force systems acting on the bodies $\left(V_{i}\right)(i=1, \ldots, 4)$ are defined as follows:

$$
\begin{aligned}
& \boldsymbol{F}_{i}^{(i)}=\boldsymbol{A}_{0, i}^{T}\left[0,0,-m_{i} g\right]^{T}, i=1,2,3, \\
& \boldsymbol{F}_{4}^{(4)}=\left[0,-F_{w} \cos \left(\delta+\theta_{b}\right), F_{w} \sin \left(\delta+\theta_{b}\right)\right]+ \\
& +\boldsymbol{A}_{0,4}^{T}\left[0,0,-m_{4} g\right]^{T} \\
& \boldsymbol{M}_{i}^{(i)}=[0,0,0]^{T}, i=1,2,3,
\end{aligned}
$$

and $\boldsymbol{M}_{4}^{(4)}$ is defined by the relation (8).

The graphs of the magnitude of the force $\boldsymbol{F}_{w}$ and projections $\lambda_{2}, \lambda_{3}$ and $\lambda_{4}$ are shown in Figs. 5, 6, 7, 8 .

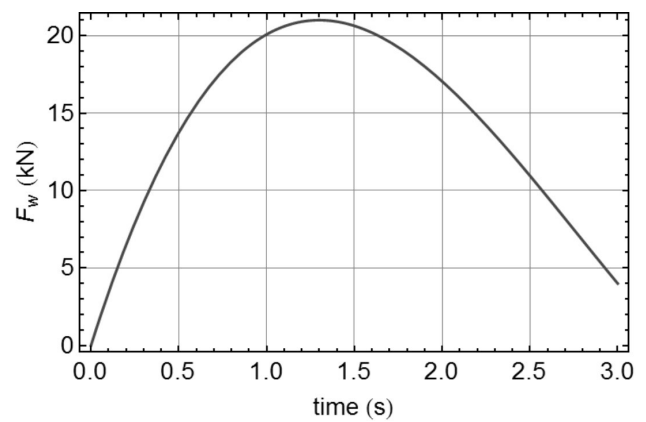

Figure 5: Magnitude of resistance digging (cutting) force $\boldsymbol{F}_{w}$ versus time

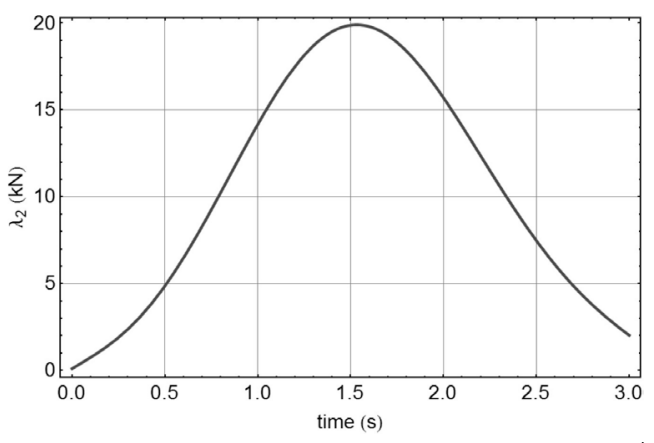

Figure 6: The projection $\lambda_{2}$ of the force $\boldsymbol{R}^{*}$ onto the axis $O^{*} \eta^{*}$

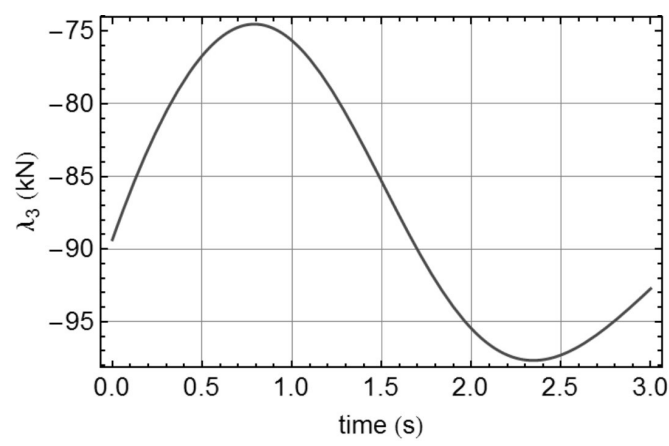

Figure 7: The projection $\lambda_{3}$ of the force $\boldsymbol{R}^{*}$ onto the axis $O^{*} \zeta^{*}$ 


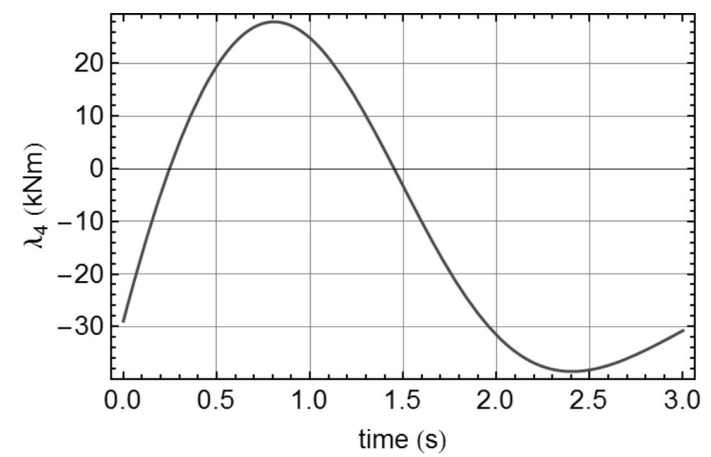

Figure 8: The projection $\lambda_{4}$ of the torque $\boldsymbol{M}^{*}$ onto the axis $O^{*} \xi^{*}$

For the considering digging transportation task, the following holds:

$$
\begin{aligned}
& \lambda_{1}(t) \equiv 0, \\
& \lambda_{5}(t) \equiv 0 .
\end{aligned}
$$

\section{CONCLUSIONS}

In this paper, expressions in symbolic form for projections of the force $\boldsymbol{R}^{*}$ and the moment of couple of forces $\boldsymbol{M}^{*}$, which is exposed to suspension ring of the underframe, are presented. These expressions allow to during the excavation phase examine the effect of various design parameters of excavator and the relevant factors in process of interaction between the bucket and the soil to the load of the suspension ring. The expressions (12) (13) can be used also in the caselifting and returning transport operations. Approach from paper [14] allows the loading of the suspension ring be determined without the need for determining reaction forces in joints $\mathrm{O}_{2}, \mathrm{O}_{3}$ и $\mathrm{O}_{4}$ , so that in meaning of computation is superior in regard to the Newton-Euler approch [15].

\section{ACKNOWLEDGEMENTS}

Support for this research was provided by the Ministry of Education, Science and Technological Development of the Republic of Serbia under Grants No. TR35006 and No. TR35038. This support is gratefully acknowledged.

\section{REFERENCES}

[1] S.Šalinić, G.Bošković, M.Nikolić, "Dynamic modelling of hydraulic excavator motion using Kane's equations," Automation in Construction, Vol. 44, pp. 56$62,(2014)$
[2] A.A.Shabana, "Dynamics of Multibody systems, Validation," third ed. Cambridge University Press, New York, 2005.

[3] V. Čović, M. Lazarević, "Mechanics of Robots," Faculty of Mechanical Engineering of Belgrade, Belgrade, 2009. (in Serbian)

[4] P. Nikravesh, "Computer-aided Analysis of Mechanical Systems," Prentice Hall, Englewood Cliffs, New Jersey, 1988.

[5] J. Angeles, O. Ma, "An algorithm for the inverse dynamics of n-axis general manipulators using Kane's equations," Comput. Math. Appl. 17 (1989) 1545-1561.

[6] J. Angeles, "Faundamentals of Robotic Systems: Theory, Methods, and Algorithms," $3^{\text {rd }}$ ed., SpringerVerlag, New York, 2007.

[7] A.J. Koivo, M. Thoma, E. Kocaoglan, J. AndradeCetto, "Modeling and control of excavator dynamics during digging operation," J. Aerosp. Eng.9 (1996) 10-18.

[8] T.V. Alekseeva, K.A. Artem'ev, A. Bromberg, "Machines for Earth Moving Work: Theory and Calculations," A. A. Balkema, Rotterdam, 1992.

[9] H. Cannon, S. Singh, "Models for automated earthmoving," Experimental Robotics VI, Lecture Notes in Control and Information Sciences, vol. 250, Springer Verlag, 2000, pp. 163-172.

[10] S.P. DiMaio, S.E. Salcudean, C. Reboulet, S. Tafazoli, K. Hashtrudi-Zaad, "A virtual excavator for controller development and evaluation," Proceedings of the 1998 IEEE International Conference on Robotics and Automation, Leuven, Belgium, vol. 1, 1998, pp. 52-58.

[11] P.K. Vähä, A.J. Koivo, M.J. Skibniewski, "Excavation dynamics and effects of soil in digging," Proceedings of the 8th International Symposium on Automation and Robotics in Constructions, ISARC-8, Stuttgart, Germany, 1991, pp. 297-306.

[12] P.K. Vähä, M.J. Skibniewski, "Dynamic model of excavator,” J. Aerosp. Eng. 6 (1993) 148-158

[13] Z. Towarek, Dynamics of a single-bucket excavator on a deformable soil foundation during the digging of ground, Int. J. Mech. Sci. 45 (2003) 1053-1076.

[14] S. Šalinić, "Determination of joint reaction forces in a symbolic form in rigid multibody systems," Mech. Mach. Theory 46 (2011) 1796-1810

[15] J.Y.S. Luh, M.W. Walker, R.P.C. Paul, "On-line computational scheme for mechanical manipulators," Transactions of ASME, Journal of Dynamic Systems, Measurement, and Control 102 (1980) 69-76. 